\title{
Contribución al conocimiento de cianobacterias de dos embalses de la provincia de Córdoba, Argentina
}

\author{
Contribution to the Knowledge of Cyanobacteria of two Reservoirs \\ of the Province of Córdoba, Argentina
}

\author{
Daga, Claudia ${ }^{1,2 *}$; Raquel Murialdo2; María C. Fernández Belmonte ${ }^{3}$ \\ 1 Cátedra de Diversidad Biológica I. Facultad de Ciencias Exactas Físicas y Naturales. Universidad Na- \\ cional de Córdoba. Argentina. Avenida Vélez Sarsfield 299, (5000) Córdoba Argentina. \\ 2 Área de Proyectos Especiales. Facultad de Ciencias Exactas Físicas y Naturales. Universidad Nacional \\ de Córdoba. Argentina. Avenida. Vélez Sarsfield 299, (5000) Córdoba Argentina. \\ 3 Cátedra de Morfología Vegetal. Departamento. Ciencias Agropecuarias. Facultad de Ingeniería y Cien- \\ cias Agropecuarias, Universidad Nacional de San Luis. Argentina. \\ * Autor corresponsal: inesclaudiadaga@gmail.com
}

\begin{abstract}
Resumen - Daga, Claudia; Raquel Murialdo; María C. Fernández Belmonte. 2017. "Contribución al conocimiento de cianobacterias de dos embalses de la provincia de Córdoba, Argentina". Lilloa 54 (2). El presente trabajo constituye un aporte al conocimiento de las cianobacterias presentes en dos embalses de la provincia de Córdoba, San Roque y La Quebrada. Se realizaron muestreos estacionales en los embalses y desembocaduras de tributarios desde febrero de 2014 a febrero de 2016. Para la extracción de las muestras se utilizó un muestreador tipo Van Dorn y una red de plancton de $20 \mu \mathrm{m}$ de poro. Se midieron parámetros físicos y químicos in situ y las muestras fueron observadas in vivo con microscopio binocular. Se identificaron 37 especies de cianobacterias, Synechococcales (15); Oscillatoriales; (12); Chroococcales (7); Spirulinales (2) y Nostocales (1). De las especies citadas 17 son nuevas para la provincia de Córdoba, de las cuales cuatro son productoras de toxinas.
\end{abstract}

Palabras clave: Cyanobacteria, embalses, Argentina.

- Abstract - Daga, Claudia; Raquel Murialdo; María C. Fernández Belmonte. 2017. "Contribution to the knowledge of Cyanobacteria of two reservoirs of the province of Córdoba, Argentina". Lilloa 54 (2). The present work constitutes a contribution to the knowledge of the Cyanobacteria present in two reservoirs of the province of Córdoba, San Roque and The Quebrada. Seasonal samplings were carried out in the reservoirs and tributary mouths from February 2014 to February 2016. A Van Dorn sampler and 20 im pore plankton net were used to extract the samples. Physical and chemical parameters were measured in situ and the samples were observed in vivo with a binocular microscope. We identified 37 taxa belonging to the orders: Synechococcales (15); Oscillatoriales (12); Chroococcales (7); Spirulinales (2) and Nostocales (1). Of the species cited 17 are new records for the province of Córdoba and four are producing toxins.

Keywords: Cyanobacteria, reservoirs, Argentina.

\section{INTRODUCCIÓN}

La provincia de Córdoba está ubicada en el centro del territorio argentino, pertenece a una región semiárida, las lluvias se concentran en el verano y a principio de otoño con una distribución irregular siendo abundantes en el sudeste (donde provocan periódi-

Recibido: 07/07/17 - Aceptado: 01/11/17 camente inundaciones de campos) y escasas en el noroeste provincial (región afectada por sequías prolongadas). Debido a estas características Córdoba es una de las regiones donde se han construido obras hidráulicas para el aprovechamiento de los recursos hídricos. La construcción de presas comenzó a finales del siglo XIX y tuvo un fuerte impulso a mediados del siglo XX con la finalidad de regular crecidas, proveer agua para consumo 
humano, riego y en menor medida, con el propósito de generar energía eléctrica. En la actualidad cuenta con veinte presas de las cuales algunas de ellas sufren el deterioro constante de la calidad de sus aguas, interfiriendo así con los usos para los cuales fueron creadas (Reyna et al., 2005).

Los sistemas leníticos considerados representan importantes reservorios de agua. El embalse San Roque fue el primer dique de América del Sur (1944) y abastece de agua potable aproximadamente al $70 \%$ de la población de la ciudad de Córdoba.

El embalse La Quebrada se terminó de construir en 1975 y provee de agua potable a la ciudad de Río Ceballos y otras localidades serranas. El crecimiento demográfico desordenado, que ha experimentado la zona en las últimas décadas, unido a la falta de un adecuado manejo ambiental de los recursos hídricos, ha llevado a la degradación de estos ambientes leníticos, especialmente por el aporte excesivo de nutrientes orgánico de origen variado, lo que favorece los procesos de eutrofización. Como consecuencia de esto son frecuentes las floraciones de dinoflagelados y de cianobacterias (Bonetto, 1976; Gavilán, 1981; Granero et al., 2002; Rodríguez et al., 2010; Pierotto y Daga, 2013; Daga y Pierotto, 2011). El embalse San Roque es el cuerpo de agua más comprometido, presentando elevados niveles de eutrofia con reportes de florecimientos de cianobacterias productoras de toxinas como Microcystis aeruginosa (Pizzolón et al., 1999). Estudios realizados, en este mismo embalse, por Ame et al. (2000) donde analizaron por HPLC muestras de 30 floraciones ocurridos entre 1998 y 2001, reconocieron dos variedades de microcistinas y concluyeron que el 96\% de las floraciones estudiadas presentaban toxinas.

El dique La Quebrada es uno de los más nuevos; durante el verano experimenta procesos de eutrofización con el desarrollo desmedido de blooms algales, principalmente de cianobacterias y dinoflagelados (Daga y Pierotto, 2014). El objetivo de este trabajo es contribuir al conocimiento de la riqueza florística de cianobacterias, aportando información sobre los nuevos taxones encontrados.

\section{MATERIALES Y MÉTODOS}

El área de estudio se halla comprendida aproximadamente entre los meridianos $64^{\circ}$ $20^{\prime}$ y $64^{\circ} 27^{\prime}$ de Longitud Oeste y entre los paralelos $31^{\circ} 14^{\prime}$ y $31^{\circ} 22^{\prime}$ de Latitud Sur (Fig. 1). Los sistemas hídricos forman parte de la cuenca del río Suquía (Primero) y pertenece al gran sistema endorreico que tiene como nivel de base la Laguna Mar Chiquita. El clima en la zona es templado, la temperatura puede alcanzar valores bajo cero en invierno y en el verano puede superar los $40^{\circ} \mathrm{C}$. La cuenca está caracterizada por una precipitación media anual de 650-700 $\mathrm{mm}$ donde se alternan años secos y años lluviosos.

Se realizaron muestreos estacionales, comprendidos entre enero de 2014 y febrero de 2016. Los sitios analizados correspondieron a: embalse La Quebrada: E. 1: estación cierre embalse La Quebrada; E. 2: desembocadura arroyo Colanchanga; E. 3: desembocadura arroyo Los Hornillos. Embalse San Roque, E. 1: estación cierre embalse San Roque; E. 2: desembocadura río Cosquín; E. 3: desembocadura arroyo Las Mojarras; E. 4: desembocadura arroyo Los Chorrillos; E. 5: desembocadura río San Antonio. Las muestras de cianobacterias se tomaron en los cierres de ambos embalses con un muestreador tipo Van Dorn a los 0,30 metros y en la desembocadura de los tributarios se recolectaron mediante red de plancton (20 $\mu \mathrm{m}$ de diámetro de poro) para la concentración de las mismas. Se filtró un volumen conocido de agua (10 litros) y se colocó en botellas para su posterior análisis.

En cada una de las estaciones de muestreo, se realizaron mediciones «in situ» de variables físicas y químicas: $\mathrm{pH}$ (Medidor digital de pH WATERPROOF), temperatura y conductividad eléctrica (Instrumental digital de conductividad/temperatura WATERPROOF), oxígeno disuelto (Medidor digital de oxigeno disuelto OAKTON). En el paredón de cierre de cada embalse se midió la trans- 


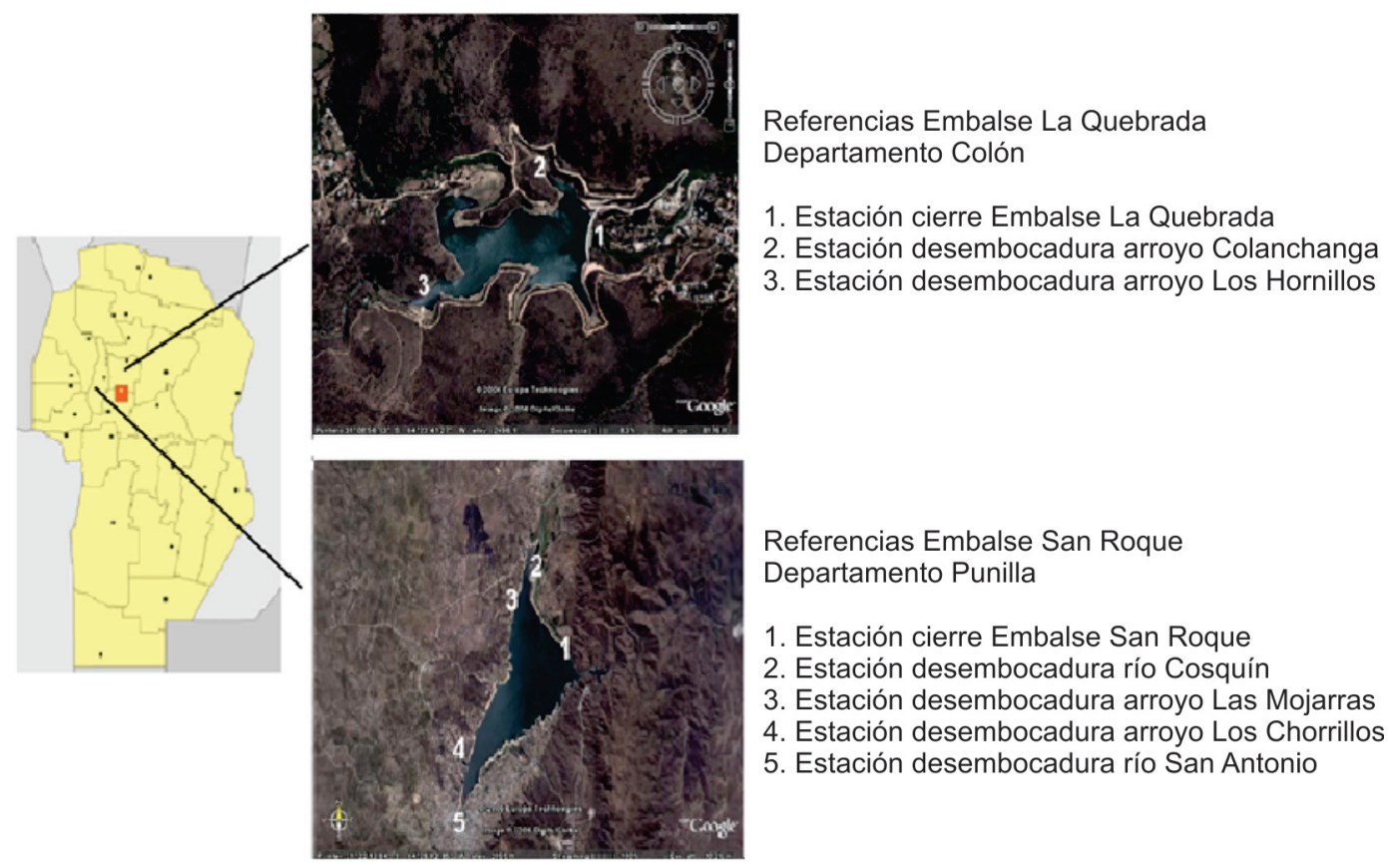

Fig. 1. Áreas de estudio: Embalses San Roque y La Quebrada. Se detallan las estaciones de muestreo.

parencia del agua con el Disco de Secchi. El análisis taxonómico se realizó mediante el empleo de microscopio óptico marca Kyowa (mod. LSCB-VC-2B-L), las muestras fueron observadas en vivo con un aumento de 40x y 100x. Se conservaron con formaldehído (2- 4\%) e incorporaron al herbario del Laboratorio de Hidrobiología bajo la sigla LH, UNC. El ordenamiento sistemático se realizó de acuerdo a Komárek et al., 2014 y para las determinaciones de las especies se utilizaron los trabajos de Komárek y Anagnostidis (1998, 2005), Komárek (2013) y se consultó AlgaeBase (2017).

\section{RESULTADOS Y DISCUSIÓN}

En la Tabla 1 se observan los valores medios y desvío estándar de los resultados estacionales obtenidos en el período estudiado, para los parámetros físicos y químicos en embalse San Roque. La transparencia del agua, varió entre $0,90 \mathrm{~m}$ en verano $\mathrm{y}$ $1,20 \mathrm{~m}$ en invierno; para embalses de regiones semiáridas, la baja transparencia de Secchi, con valores por debajo de 1,2 m estaría relacionada con altas concentraciones de material en suspensión como resultado de la erosión de sus extensas cuencas suma-

Tabla 1. Valores medios y desvío estándar estacionales, del período estudiado de parámetros físicos y químicos en el embalse San Roque.

\begin{tabular}{l|lllll}
\hline \multicolumn{1}{c|}{$\begin{array}{c}\text { Parámetros } \\
\text { Estaciones }\end{array}$} & Transparencia $(\mathrm{m})$ & Temperatura & $\mathrm{CE}\left(\mu \mathrm{S} . \mathrm{cm}^{-1}\right)$ & $\mathrm{pH}$ & $\mathrm{OD}\left(\mathrm{mg} \cdot \mathrm{L}^{-1}\right)$ \\
\hline Otoño & $1,10+/-0,03$ & $15,48+/-0,71$ & $212.87+/-36.77$ & $8,07+/-8,07$ & $7,63+/-3,10$ \\
Invierno & $1,20+/-0,03$ & $15,35+/-2,12$ & $289,56+/-66,47$ & $8,59+/-0,28$ & $8,53+/-1,69$ \\
Primavera & $1,11+/-0,28$ & $24+/-1,41$ & $277,98+/-2,83$ & $9,25+/-0,21$ & $5,67+/-1,42$ \\
Verano & $0,90+/-0,35$ & $27+/-1,41$ & $222,24+/-18,38$ & $9,00+/-0,07$ & $3,94+/-1,43$ \\
\hline
\end{tabular}

Nota: T. Temperatura, C E: Conductividad Eléctrica, OD: Oxígeno Disuelto 
Tabla 2. Valores medios y desvío estándar estacionales, del período estudiado de parámetros físicos y químicos en el embalse La Quebrada.

\begin{tabular}{l|lllll}
\hline $\begin{array}{l}\text { Parámetros } \\
\text { Estaciones }\end{array}$ & Transparencia $(\mathrm{m})$ & Temperatura & $\mathrm{CE}\left(\mu \mathrm{s} . \mathrm{cm}^{-1}\right)$ & $\mathrm{pH}$ & $\mathrm{OD}\left(\mathrm{mg} . \mathrm{L}^{-1}\right)$ \\
\hline Otoño & $1,49+/-0,14$ & $13,04+/-3,54$ & $304,47+/-43,13$ & $8,40+/-0,14$ & $10,18+/-1,38$ \\
Invierno & $1,71+/-0,35$ & $20,00+/-0,35$ & $302,6+/-15,56$ & $8,99+/-0,28$ & $6,61+/-2,05$ \\
Primavera & $1,24+/-0,14$ & $24,00+/-1,41$ & $292,4+/-7,78$ & $9,05+/-0,21$ & $6,45+/-0,56$ \\
Verano & $1,40+/-0,03$ & $25,41+/-2,12$ & $295,73+/-36,77$ & $8,68+/-0,57$ & $10,09+/-0,16$ \\
\hline
\end{tabular}

Nota: T. Temperatura, C E: Conductividad Eléctrica, OD: Oxígeno Disuelto

do al componente fitoplanctónico del agua (Quirós, 2002). En tanto la temperatura del agua alcanzó su máximo valor $\left(27^{\circ} \mathrm{C}\right)$ en verano y el mínimo $\left(15^{\circ} \mathrm{C}\right)$ en invierno. $\mathrm{El} \mathrm{pH}$ del agua presentó-registros mayores a 8,0. La conductividad en el embalse San Roque tuvo su máximo valor medio en invierno $\left(289,56 \mu \mathrm{S} \mathrm{cm}^{-1}\right)$ y el menor en la primavera $\left(212,87 \mu \mathrm{S} . \mathrm{cm}^{-1}\right)$. Durante el período invernal la ausencia de precipitaciones concentra los iones disueltos aumentando de esta manera la conductividad, mientras que en períodos de lluvia ocurre el proceso inverso, producto de la dilución (Rodríguez et al. 2002). La menor concentración de oxígeno disuelto fue de $3,94 \mathrm{mg} \cdot \mathrm{L}^{-1}$ (verano) y el máximo fue de $8,23 \mathrm{mg} \cdot \mathrm{L}^{-1}$ (otoño), este parámetro es dependiente de la temperatura; aguas más cálidas son capaces de disolver menores cantidades de oxígeno. En tanto el consumo de oxígeno depende de la respiración y descomposición de materia orgánica. En relación a esto se pudo observar que al final del verano comienza la degradación de organismos del fitoplancton en especial de Ceratium sp. generando situaciones de anoxia. Se ha demostrado que valores inferiores a $4 \mathrm{mg} \cdot \mathrm{L}^{-1}$ es considerado crítico para la supervivencia de peces (Rodríguez et al. 2013).

Respecto al embalse La Quebrada en la Tabla 2 se observan los registros medios y desvíos estándar de los parámetros físicos y químicos. La transparencia, osciló entre 1,24 $\mathrm{m}$ en primavera y $1,71 \mathrm{~m}$ en invierno. La temperatura del agua fue menor en otoño con una mínima de $13,04^{\circ} \mathrm{C}$ y las máximas con $25,41^{\circ} \mathrm{C}$ en verano. El $\mathrm{pH}$ del agua fue alcalino, siempre superior a 8,04 y la conductividad varió entre 292,4 y 304,47 $\mu$ S.cm1. El oxígeno disuelto presentó los valores máximos en verano con 10,09 mg. $\mathrm{L}^{-1}$ y los mínimos en primavera con $6,45 \mathrm{mg} \cdot \mathrm{L}^{-1}$.

La riqueza específica en el embalse San Roque fue mayor en la desembocadura del río San Antonio (Fig. 2) la misma situación fue observada por Rodríguez et al. (2013). En el embalse La Quebrada en la desembocadura del arroyo Los Hornillos es donde se

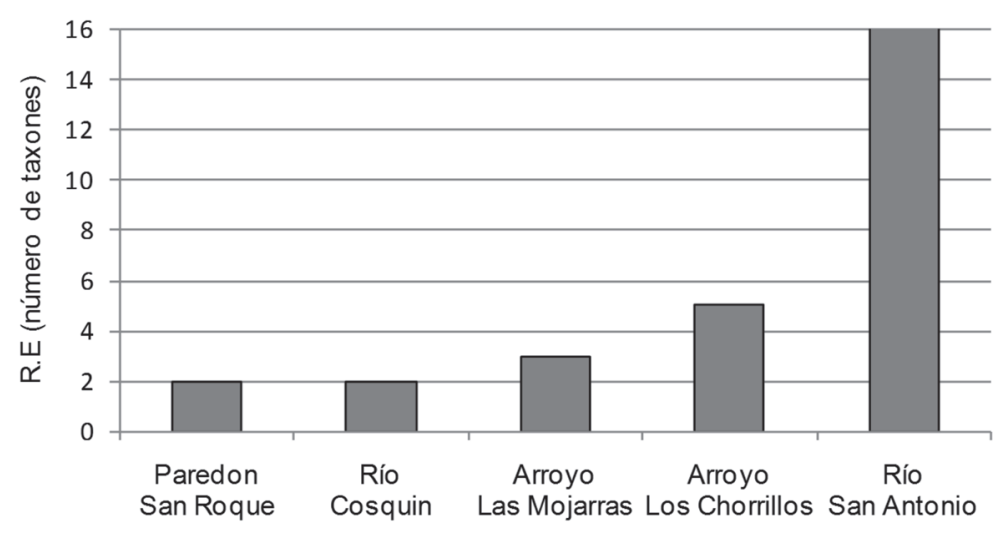

Fig. 2. Variación de la Riqueza Específica (RE) de cianobacterias en el embalse San Roque. 


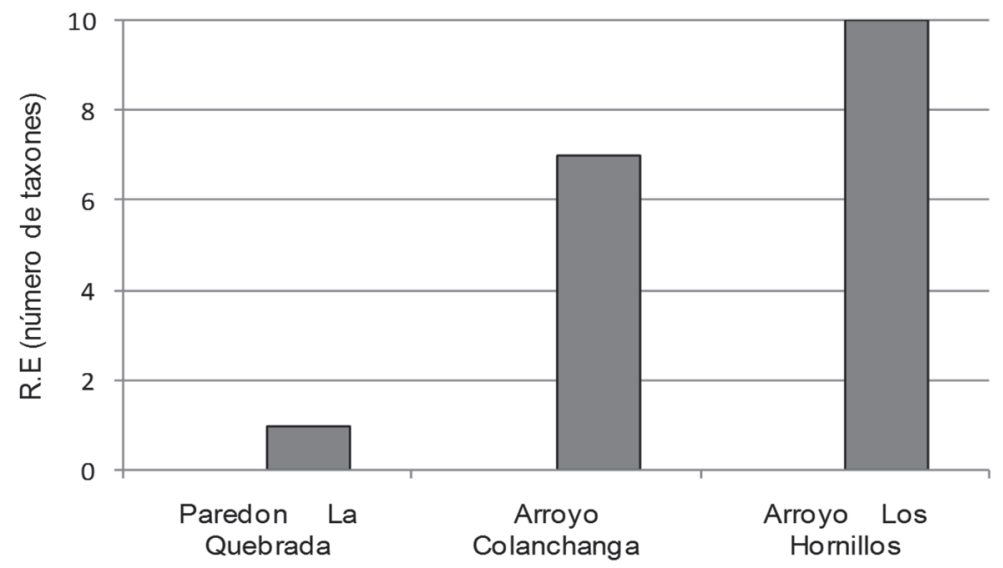

Fig. 3. Variación de la Riqueza Específica (RE) de cianobacterias en el embalse La Quebrada.

observó mayor riqueza de cianobacterias. (Fig. 3).

A continuación se describen las 17 especies consideradas nuevas citas para la provincia de Córdoba (E.S.R: embalse San Roque; E.L.Q: embalse La Quebrada). Además se agregan medidas morfométricas y algunos datos de la autoecología de los nuevos registros de taxones.

\section{ORDEN CHROOCOCCALES}

\section{Aphanothece floccosa}

(Zalessky) Cronberg et Komárek, 1944

(Fig. 4 A)

Colonias microscópicas, más o menos esféricas a ovales, células agregadas densamente, alargadas de coloración verdosa, sin aerótopos.

Dimensiones. - Diámetro de la colonia: 50-400 $\mu \mathrm{m}$; largo de las células: 3-4 $\mu \mathrm{m}$; ancho de las células: 1-2 $\mu \mathrm{m}$.

Distribución y hábitat.- Nueva cita para Córdoba. Epipélica, metafítica. Especie citada para lagos oligotróficos a mesotróficos (Komárek \& Anagnostidis, 1998).

Material estudiado.- ARGENTINA. Prov. Córdoba, Dpto. Colón, embalse La Quebrada, 14-XI-2015, C. Daga 183 (L. H.UNC).

\author{
Aphanothece smithii \\ Komárková-Legnerová \\ \& G.Cronberg, 1994 (Fig. 4 B)
}

Colonias microscópicas, esféricas, alargadas a irregulares. Células ovales a cilíndricas de coloración verde azulado con contenido más o menos homogéneo, unidas por un mucílago incoloro.

Dimensiones celulares. - Largo: 4,2 $\mu \mathrm{m}$; ancho: $2 \mu \mathrm{m}$.

Distribución y hábitat. - Citada para Neuquén (Wenzel y Díaz, 2008). Nueva cita para Córdoba. Planctónica, de ambientes leníticos en desembocadura del arroyo Los Hornillos.

Material estudiado. - ARGENTINA. Prov. Córdoba, Dpto. Colón, embalse La Quebrada, 4-XI-2015, C. Daga 183 (L. H. UNC).

Chondrocystis cf. sarcinoides (Elenkin) Komárek et Anagnostidis, 1995

(Fig. 4 C)

Subcolonias ligeramente aplanadas de coloración verde azulado, com mucílago incoloro. Células esféricas.

Dimensiones. - diámetro de las subcolonias: $40 \mu \mathrm{m}$; diámetro de la célula: $3,5 \mu \mathrm{m}$.

Distribución y hábitat. - Nueva cita para la provincia de Córdoba. Planctónica. 
Observaciones.- No se encontraron las colonias macroscópicas, solo se identificaron las subcolonias.

Material estudiado.- ARGENTINA. Prov. Córdoba, Dpto. Colón, embalse La Quebrada, 4-XI-2015, C. Daga 183 (L.H.UNC).

\section{ORDEN SyNECHOCOCCALES}

\section{Leptolyngbya notata (Schmidle) Anagnostidis et Komárek, 1988}

(Fig. 4 G)

Filamentos curvados con falsas ramificaciones, con vaina delgada e incolora. Tricomas verde azulado pálido, células cilíndricas de 2 a 3 veces más largas que anchas, célula apical redondeada. Pared sin constricción.

Dimensiones celulares. - largo: 4-6 $\mu \mathrm{m}$; ancho: $2 \mu \mathrm{m}$.

Distribución y hábitat. - Citada para Buenos Aires (Tell, 1985); Neuquén (Wenzel \& Díaz, 2008). Nueva cita para la provincia de Córdoba. Material recolectado en la desembocadura de Arroyo Colanchanga y Los Chorrillos, se desarrolla como epífita sobre Chaetomorpha sp., en época invernal. Especie edafofítica y menos frecuente en el agua dulce (Komárek et al., 2005).

Material estudiado. - ARGENTINA. Prov. Córdoba, Dpto. Colón, embalse La Quebrada, 25-VIII-2015, C. Daga 172 (L. H. UNC); Dpto. Punilla, embalse San Roque, 1-IX2015, C. Daga 175 (L.H.UNC); 25-XI-2015, C. Daga 186 (L.H.UNC).

\section{Leptolyngbya ochraceae \\ (Thuret ex Gomont) Anagnostidis et Komárek, 1988}

(Fig. 4 I)

Filamentos generalmente rectos a poco curvados. Tricomas verdes azulados, con vaina de coloración ocre o pardo amarillenta, célula apical redondeada.

Dimensiones del filamento.- largo: 160$800 \mu \mathrm{m}$; ancho: 1,6-2 $\mu \mathrm{m}$.

Distribución y hábitat. - Citada para la provincia de Corrientes (Tell, 1985).Planctónica, cosmopolita. Nueva cita para la pro- vincia de Córdoba. Es frecuente encontrarla en las márgenes de arroyos y lagos, cuando está en abundancia le otorga coloración ocre al agua.

Material estudiado. - ARGENTINA. Prov. Córdoba, Dpto. Colón, embalse La Quebrada, 07-V-2015, C. Daga 160 (L H. UNC).

\section{Limnothrix redekei \\ (Van Goor) Meffert, 1988}

(Fig. 4 D)

Tricomas solitarios rectos a levemente arqueados, pared celular en algunos casos con constricciones leves. Células de coloración verde pálido con aerotopos refringentes en los extremos de las células. Célula apical redondeada o algo cónica con una vacuola.

Dimensiones celulares. - largo: 7-8 $\mu \mathrm{m}$; ancho: $2-2,5 \mu \mathrm{m}$.

Distribución y hábitat.- Nueva cita para Córdoba. Planctónica. Especie productora de toxinas, lipopolisacaridos (LPS). Citada para ambientes de mesotrófico a eutrófico, común en zonas templadas a frías. En Uruguay ha sido citada tanto en invierno como en verano (Bonilla, 2009).

Material estudiado. - ARGENTINA. Prov. Córdoba, Dpto. Colón, embalse La Quebrada, 14-XI-2015, C. Daga 183 (L. H.UNC).

\section{Limnothrix mirabilis \\ (Böcher) Anagnostidis, 2001}

(Fig. 4 E)

Tricomas solitarios rectos a levemente arqueados, con movilidad. Pared celular sin constricción, vaina ausente. Células con contenido granular, célula apical redondeada.

Dimensiones. - largo de los tricomas: 250-300 $\mu \mathrm{m}$; largo de las células: $4 \mu \mathrm{m}$; ancho de la célula: $2 \mu \mathrm{m}$.

Distribución y hábitat. - Nueva cita para Córdoba. Planctónica.

Material estudiado.- ARGENTINA. Prov. Córdoba, Dpto. Colón, embalse La Quebrada, 14-XI-2015, C. Daga 190 (L. H.UNC). 


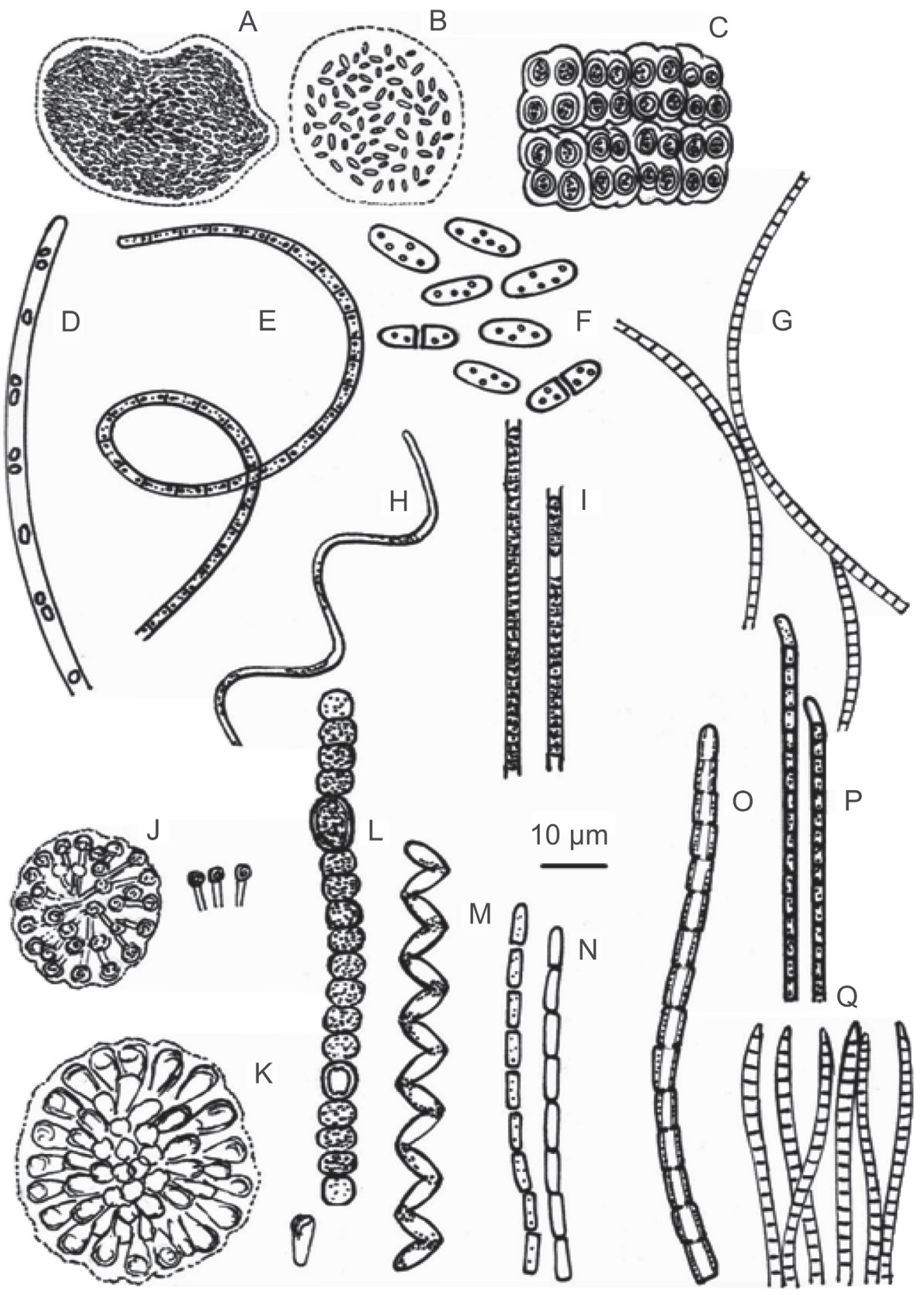

Fig. 4. A) Aphanothece floccosa. B] Aphanothece smithii. C) Chondrocystis cf. sarcinoides. D) Limnothrix redekei. E] Limnothrix mirabilis. FJ Synechococcus mundulus. G] Leptolyngbya notata. H) Glaucospira agilíssima. I) Leptolyngbya ochraceae. J) Woronichinia tener. K) Woronichinia karelica. L) Dolichospermum viguieri. M) Spirulina major. N) Pseudonabaena limnetica. 0) Pseudoanabaena galeata. P) Geitlerinema exile. Q) Phormidium corium. 


\section{Pseudoanabaena galeata Böcher, 1949}

(Fig. 4 O)

Tricomas solitarios o agregados formando matas de coloración verde azulado, generalmente rectos o poco onduladas, con constricción entre dos células contiguas. Células cilíndricas más largas que anchas, con aerotopos en los polos. Célula apical redondeada.

Dimensiones. - largo de los tricomas: 40$100 \mu \mathrm{m}$; largo de las células: 2,5-3,2 $\mu \mathrm{m}$; ancho de las células: 1,6-2,5 $\mu \mathrm{m}$.

Distribución y hábitat. - Nueva cita para Córdoba. Cosmopolita. Planctónica a bentónica, sobre rocas o vegetación a orillas de los ríos.

Material estudiado. - Argentina. Prov. Córdoba, Dpto. Punilla, embalse San Roque, 09-I-2014, C. Daga 141(L. H. UNC).

Pseudonabaena limnetica

(Lemmermann) Komárek, 1974

(Fig. $4 \mathrm{~N}$ )

Tricomas solitarios, ligeramente recto a curvo, móviles. Células largas cilíndricas, constreñidas en las zonas de contacto, de coloración verde azulado pálido, contenido homogéneo con aerótopos. Célula apical redondeada.

Dimensiones celulares. - largo: 3,5-16 $\mu \mathrm{m}$; ancho 1,5-2 $\mu \mathrm{m}$.

Distribución y hábitat. - Citada para las provincias de Buenos Aires, Corrientes, Santa Cruz, Chubut, Santa Cruz (Tell, 1985). Nueva cita para Córdoba. Cosmopolita. Planctónica a bentónica, de acuerdo a literatura puede formar floraciones y causar agotamiento de oxígeno en el agua, pero no es productora de toxinas (Chomérat et al., 2007). Se la encontró asociada a Planktothrix agardhii.

Material estudiado. - ARGENTINA. Prov. Córdoba, Dpto. Colón, embalse La Quebrada, 04-II-2016, C. Daga 194 (L. H.UNC).
Synechococcus mundulus

Skuja, 1964

(Fig. 4 F)

Células solitaria o reunidas de a dos, cilíndricas a ovales, extremos redondeados; contenido celular verde azulado, con gránulos refringentes. Las células se dividen por fisión binaria y las células hijas pueden tener distintos tamaños y permanecer unidas por un tiempo.

Dimensiones celulares. - largo: 8-12 $\mu \mathrm{m}$; ancho: $4-6 \mu \mathrm{m}$.

Distribución y hábitat.- Nueva cita para Córdoba. Bentónica, epífitas sobre otras algas.

Material estudiado.- ARGENTINA. Prov. Córdoba, Dpto. Punilla, embalse San Roque, 29-XI-2014, C. Daga 141b (L. H.UNC).

\section{Woronichinia tenera}

(Skuja) Komárek et Hindák, 1988

(Fig. $4 \mathrm{~J}$ )

Colonias pequeñas, más o menos irregulares esféricas, oval o lobadas, rodeadas por mucílago, de 8 a 100 células, más o menos esféricas a obovadas. Coloración verde azulado con contenido homogéneo.

Dimensiones. - Diámetro de la colonia: $25 \mu \mathrm{m}$; diámetro de la célula: 2-2,5 $\mu \mathrm{m}$.

Distribución y hábitat. - Nueva cita para Córdoba. Especie citada como bentónica (Komarék et al., 1998), en este estudio formó parte del plancton.

Material estudiado.- ARGENTINA. Prov. Córdoba, Dpto. Punilla, embalse San Roque, 29-XI-2014, C. Daga 156 (L. H. UNC).

\section{Woronichinia karelica}

Komárek et Komárkova-Legnerová, 1992

(Fig. $4 \mathrm{~K}$ )

Colonias solitaria, irregulares esféricas a ovales, cuando son maduras se pueden observar subcolonias. Células alargadas dispuestas radialmente en la colonia. 
Tabla 3. Especies de Cyanobacteria presentes en cada uno de los embalses estudiados. Ver: verano; Oto: otoño; Inv: invierno; Prim: primavera.

\begin{tabular}{|c|c|c|c|c|c|c|c|c|}
\hline \multirow{2}{*}{$\begin{array}{l}\text { Embalses } \\
\text { Taxones }\end{array}$} & \multicolumn{4}{|c|}{ Embalse San Roque } & \multicolumn{4}{|c|}{ Embalse La Quebrada } \\
\hline & Ver & Oto. & Inv. & Prim. & Ver. & Oto. & Inv. & Prim. \\
\hline Aphanothece floccosa & & & & & & & & $\mathrm{X}$ \\
\hline Aphanothece smithii & & & & & & & & $x$ \\
\hline Borzia trilocularis & & & & $x$ & & & & \\
\hline Chamaesiphon incrustans & & & $\mathrm{X}$ & & & & & \\
\hline f.incrustans & & & & & & & & \\
\hline Chondrocystis cf. sarcinoides & & & & & & & & $\mathrm{x}$ \\
\hline Chroococcus minutus & $x$ & & & & & & & \\
\hline Chroococcus turgidus & & $x$ & & & & & & \\
\hline Cyanothece aeruginosa & & & $\mathrm{X}$ & & & & & \\
\hline Dolichospermum viguieri & & & & $\mathrm{x}$ & & & & \\
\hline Glaucospira agilissima & $\mathrm{X}$ & & & $x$ & & & & \\
\hline Geintlerinema exile & & & & & $\mathrm{X}$ & & & \\
\hline Gomphosphaeria aponina & & $\mathrm{X}$ & & & & & & \\
\hline Leptolyngbya notata & & & & $\mathrm{X}$ & & & $\mathrm{X}$ & \\
\hline Leptolyngbya ochraceae & & & & & & & & \\
\hline Limnothrix mirabilis & $\mathrm{x}$ & & & & & & & $\mathrm{x}$ \\
\hline Limnothrix redekei & & & & & & & & $x$ \\
\hline Lyngbya aestuarii & $\mathrm{X}$ & & & & & & & \\
\hline Merismopedia convoluta & $x$ & & & & & & & \\
\hline Merismopedia elegans & & & & $x$ & & & & \\
\hline Merismopedia glauca & & & $x$ & $x$ & $\mathrm{x}$ & $x$ & & \\
\hline Microcystis aeruginosa & & $x$ & $x$ & & & & & \\
\hline Oscillatoria limosa & $\mathrm{X}$ & $x$ & $\mathrm{X}$ & $\mathrm{X}$ & & & & \\
\hline Oscillatoria tenuis & & & & & $x$ & & & \\
\hline Phormidium breve & & $\mathrm{X}$ & & & & & & \\
\hline Phormidium corium & $\mathrm{X}$ & & & & & & & \\
\hline Phormidium retzii & $\mathrm{X}$ & & & & & & & \\
\hline Phormidium simplicissimum & $x$ & & & & & & & \\
\hline Planktothrix agardhii & & & & & $X$ & & $\mathrm{X}$ & \\
\hline Pseudanabaena catenata & $x$ & & & & & & & \\
\hline Pseudoanabaena galeata & $\mathrm{X}$ & & & & & & & \\
\hline Pseudoanabaena limnetica & & & & & $\mathrm{X}$ & $\mathrm{X}$ & & \\
\hline Rhabdonema lineare & & & & & & & $\mathrm{X}$ & \\
\hline Spirulina major & $x$ & & & & & & & \\
\hline Synechococcus mundulus & $x$ & & & $x$ & & & & \\
\hline Synechocystis aquatilis & & $\mathrm{X}$ & & & & & & \\
\hline Woronichinia tenera & & & & $x$ & & & & \\
\hline Woronichinia karelica & & & & & $\mathrm{x}$ & & & \\
\hline
\end{tabular}

Dimensiones. - Diámetro de la colonia: 30-50 $\mu \mathrm{m}$; largo de las células: 3-5 $\mu \mathrm{m}$; ancho de las células: $1,5-2 \mu \mathrm{m}$.

Distribución y hábitat. - Nueva cita para la provincia de Córdoba. Planctónica.
Material estudiado.- ARGENTINA. Prov. Córdoba, Dpto. Colón, embalse La Quebrada, 23-II-2014, C. Daga 144 (L. H. UNC). 


\section{ORdEN Nostocales}

Dolichospermum viguieri

(Denis \& Frémy) Wacklin, L. Hoffmann \& Komárek, 2009

(Fig. 4 L)

Tricomas solitarios, rectos a levemente curvos con mucílago transparente o incoloro, células vegetativas esféricas o en forma de barril, ligeramente más cortas que anchas, con aerótopos. Célula apical esférica. Heterocistos esféricos, acinetos elipsoidales a ovalados.

Dimensiones celulares. - largo de celúlas vegetativas 4,5-8; diámetro de células vegetativas: 4-6 $\mu \mathrm{m}$; diámetro de los heterocistos: 4,5-6 $\mu \mathrm{m}$; largo de los acinetos: $12 \mu \mathrm{m}$; ancho: $6 \mu \mathrm{m}$.

Distribución y hábitat.- Buenos Aires (Izaguirre et al., 1990). Nueva cita para Córdoba. Planctónica, especie de ambientes eutróficos, lagos estratificados, puede ser productoras de anatoxinas-a (S) (Bonilla, 2009).

Observaciones. - El tamaño de los acinetos es menor al citado en bibliografía (Komárek y Zapomélová, 2007).

Material estudiado. - ARGENTINA. Prov. Córdoba, Dpto. Punilla, embalse San Roque, 29-XI-2014, C. Daga 156 (L. H. UNC); 28-I2016, C. Daga 191(L. H.UNC).

\section{ORDEN OSCILLATORIALES}

Geitlerinema exile

(Skuja) Anagnostidis, 1989

(Fig. 4 P)

Tricomas solitarios, rectos de coloración azul verdosa. Con constricción en la pared, células finamente granuladas, isodiamétricas más largas que anchas con los extremos atenuados y curvados movilidad intensa, los extremos se tuercen hacia el mismo lado mientras avanzan.

Dimensiones celulares. - Largo: 3,5-4 $\mu \mathrm{m}$; ancho: 3-3,5 $\mu \mathrm{m}$.
Distribución geográfica y hábitat.- Nueva cita para Córdoba. Metafítica, bentónica sobre suelo entre arenoso y lodoso.

Material estudiado.- ARGENTINA. Prov. Córdoba, Dpto. Colón, embalse La Quebrada, 04-II-2016, C. Daga 194 (L.H. UNC).

\section{Phormidium corium \\ Gomont ex Gomont, 1892}

(Fig. 4 Q)

Tricomas largos, rectos, curvos, formando colonias densamente entrecruzadas. Vaina delgada, incolora, tricomas verdes azulados, células casi isodiamétricas, dos veces más largas que anchas Célula apical redondeada, sin caliptra.

Dimensiones celulares. - Largo: $3 \mu \mathrm{m}$; ancho: 5-6 $\mu \mathrm{m}$.

Distribución y hábitat. - Citada para las provincias de Neuquén, Chubut, Santa Cruz (Tell, 1985). Nueva cita para Córdoba. Perifítica, epilítica en desembocadura del río San Antonio entre Pistia striolata y Azolla sp. También es citada para suelos cultivados (Murialdo et al., 2016).

Material estudiado.- ARGENTINA. Prov. Córdoba, Dpto. Punilla, embalse San Roque, 09-I-2014, C. Daga 141b (L. H. UNC).

\section{ORDEN SPIRULINALES}

\section{Glaucospira agilíssima Lagerheim, 1892}

(Fig. $4 \mathrm{H}$ )

Tricomas solitarios (o agrupados), espiralados, delgados y sin vaina, no atenuados en los extremos y sin constricciones; células de coloración azul verdoso pálido. Provistos de gran movilidad.

Dimensiones. - Largo de los tricomas: 50-120 $\mu \mathrm{m}$; ancho de las células: 1,5-2 $\mu \mathrm{m}$; distancia entre cada cresta: $8 \mu \mathrm{m}$.

Distribución y hábitat.- Nueva cita para Córdoba. Se la encontró en desembocadura de ríos entre macrófitas o asociada a Phormidium simplicissimun, con temperaturas de 28 y $29^{\circ} \mathrm{C}$ y pH entre 8,7 y 9,4 . 
Material estudiado.- ARGENTINA. Prov. Córdoba, Dpto. Punilla, embalse San Roque, 29-XI-2014, C. Daga 156 (Laboratorio de Hidrobiología, UNC); 28-I-2016, C. Daga 191(L.H.UNC).

\section{Spirulina major \\ Kützing ex Gomont, 1892}

(Fig. 4 M)

Tricomas solitarios, enrollados, verde azulados, generalmente rectos a poco curvados, célula apical redondeada.

Dimensiones. - Largo de los tricomas: 300-500 $\mu \mathrm{m}$; ancho de las células: $2-4 \mu \mathrm{m}$, distancia entre cada espira: $2,5-4 \mu \mathrm{m}$.

Distribución y hábitat. - Citada para Buenos Aires, San Luis, Santa Cruz (Tell, 1985); Jujuy (Mirande y Tracanna, 2007); Noroeste Argentino (Mirande y Tracanna, 2015); Tucumán (Tracanna, 1985, como Oscillatoria oscillarioides); Nueva cita para Córdoba. Cosmopolita. Se la encontró en desembocadura de ríos asociada a otras cianobacterias como Phormidium simplicissimun.

Material estudiado. - ARGENTINA. Prov. Córdoba, Dpto. Punilla, embalse San Roque, 28-I-2016, C. Daga 191(L. H. UNC).

Las principales toxinas de las cianobacterias corresponden a neurotoxinas y hepatotoxinas. Son producidas principalmente por los géneros Anabaena, Microcystis, Oscillatoria, Aphanizomenon y Lyngbya (Carmichael, 1994; Roset et. al., 2001).

\section{CONCLUSIONES}

Se determinaron treinta y siete (37) taxones de las cuales 26 se encontraron en el embalse San Roque y 13 en el embalse La Quebrada. Del total de especies identificadas 17 son nuevas citas para la provincia de Córdoba y el orden más representativo corresponde a las Synechococcales (13).

Se identificaron organismos productores de toxinas: Microcystis aeruginosa, Planktothrix agardhii, Dolichospermum viguieri, Oscillatoria limosa y especies de los géneros Gomphosphaeria, Lyngbya, Phormidium, Pseudoanabaena y Synechocystis.
La desembocadura del río San Antonio presentó el valor más alto de riqueza de especies (16). En este punto se identifico a $M$. aeruginosa, en el análisis cualitativo, se aclara que no se observaron colonias macroscópicamente. El rio San Antonio es la estación de muestreo con mayor influencia antrópica, muy próximo a su desembocadura se encuentra la ciudad de Carlos Paz de la cual el rio es receptor de desagües pluviales y escorrentías urbanas que aportan nutrientes al agua favoreciendo el crecimiento de cianobacterias, algas y macrófitas.

En el período estudiado no se registraron floraciones de cianobacterias, esta situación podría deberse a las precipitaciones ocurridas en las cuencas estudiadas (300 mm superior al promedio histórico de la región) provocando mayor turbulencia y elevada tasa de renovación del agua, condiciones que hacen poco favorables el desarrollo de floraciones.

Además de las cianobacterias encontradas en este estudio se observó también la presencia de otros grupos algales, siendo dominantes para ambos embalses diatomeas (Aulacoseira granulata y Cyclotella meneghiniana) y un dinoflagelado (Ceratium furcoides).

En el caso de Ceratium furcoides, especie no productora de toxinas, si puede originar problemas en los ecosistemas naturales; en el período de envejecimiento y muerte de las células, generan fuerte olor y anoxia para las comunidades peces. Por otra parte grandes concentraciones, entorpecen el tratamiento del agua destinada al consumo humano.

En conclusión, el embalse San Roque se encuentra con mayor grado de riesgo, en cuanto a la calidad de sus aguas, que el embalse La Quebrada. Ello se puede inferir del estudio realizado, considerando las características fisicoquímicas y la presencia de especies de cianobacterias productoras de floraciones y toxinas.

Con este trabajo se incrementa el conocimiento de la riqueza de cianobacterias para Córdoba, contribuyendo a ampliar la biodiversidad y distribución de este importante grupo de microalgas. 


\section{BIBLIOGRAFIA}

AlgaeBase. http://www. algaebase.org. (Consulta febrero-junio 2017).

Ame V., Wunderlin D., Pflugmacher S. 2000. Aislamiento e Identificación de Toxinas de Cianobacterias en el Lago San Roque (Pcia. de Córdoba). XXIII Congreso Argentino de Química - AQA, Corrientes. Argentina.

Bonetto A., Di Persia D. H., Maglianesi R, Corigliano C. 1976. Caracteres limnológicos de algunos lagos eutróficos de embalse de la Región Central Argentina. Ecosur 3 (5): 47-120.

Bonilla S. 2009. Cianobacterias planctónicas del Uruguay. Manual para la identificación y medidas de monitoreo. Montevideo: UNESCO. PHI-VII (Documento técnico, 16). ISBN: 978-92-9089-138-3.

Carmichael W. W. 1994. The toxins of cyanobacteria. Scientific American 270 (1): 78-86.

Chomérat N., Garnier R., Bertrand C., Cazaubon A. 2007. Seasonal succession of cyanoprokaryotes in a hypertrophic oligo-mesohaline lagoon from the South of France. Estuarine, Coastal and Shelf Science 72: 591-602.

Daga C., Pierotto M. 2014. Variaciones espaciales y temporales de organismos autótrofos en el embalse La Quebrada (Córdoba, Argentina). Arnaldoa 21 (1): 189-196.

Daga C., Pierotto M. 2011. Cianobacterias de la cuenca del Embalse San Roque. Boletin de la Sociedad Argentina de Botanica 46 (3-4): 227-234.

Gavilán J. G. 1981. Study water quality in the San Roque reservoir. Water Quality Bulletin Environment Canada 6 (4): 136-158.

Granero M., Bustamante M. A., Ruiz M. 2002. Hypolimnion Water Quality in an Eutrophicated Water Body: San Roque Reservoir (Córdoba, Argentina). Proceedings of 4th International Conference on Reservoir Limnology and Water Quality. Academia de ciencias de República Checa: 106-109.

Izaguirre I., Del Giorgio P., O’Farrel I., Tell G. 1990. Clasificación de 20 cuerpos de agua andino-patagónicos (Argentina) en base a la estructura del fitoplancton estival. Cryptogamie Algologie 11:31-46.

Komárek, J. 2013. Cyanoprokaryota. 3. Heterocytous Genera. In: Büdel, B.; Gärtner, G.; Krienitz, L. \& Schagerl, M. (eds): Süswasserflora von Mitteleuropa (Fresh- water Flora of Central Europe) 19/3. Springer Spektrum, Berlin. Heidelberg.

Komárek J., Anagnostidis K. 1998. Cyanoprokaryota. 1. Chroococcales. In: Ettl, h., G. Gärtner, H. Heynig \& D. Mollenhauer [eds.), Süâwasserflora von Mitteleuropa. 19/1. Gustav Fischer, Jena-Stuttgart-Lübeck-Ulm.

Komárek J., Anagnostidis K. 2005. Cyanoprokaryota. 2. Oscillatoriales. In: Büdel, B., L. Krienitz, G. Gärtner \& M. Schagerl (eds.), Süâwasserflora von Mitteleuropa.19/2. Elsevier/Spektrum Heidelberg.

Komárek J., Zapomélová E. 2007. Plankticmorphospecies of the cyanobacterial genus Anabaena $=$ subg. Dolichospermum -1. Part: coiled types. Fottea, Olomouc, 7 (1): 1- 31

Komárek J., Kastovsk J., Mares J., Johansen J. R. 2014. Taxonomic classification of cyanoprokaryotes (cyanobacterial genera) 2014, using a polyphasic approach. Preslia 86: 295-335.

Mirande V., Tracanna B. C. 2007. Diversidad de cianobacterias, clorofitas y euglenofitas en humedales de altura (Jujuy, Argentina). Lilloa 44 (1-2): 39-59.

Mirande V., Tracanna B. C. 2015. Cianobacterias de humedales de altura del Noroeste Argentino. Lilloa 52 (2): 122- 141.

Murialdo R., Fernández Belmonte C., González C., Candela R., Daga C., Gudiño G. 2016. Desarrollo de cianobacterias en un suelo agrícola de la provincia de Córdoba, en relación a la influencia de la fertilización nitrogenada y de la degradación de la atrazina. Actas VI Congreso Argentino de la SETAC. Córdoba, Argentina.

Pierotto M., Daga C. 2013. Evolución trófica del embalse La Quebrada. Actas XXIV Congreso Nacional del Agua. San Juan. Argentina.

Pizzolon L., Tracanna B., Prosperi C., Guerrero J. 1999. Cianobacterial blooms in argentinean inland water. Lakes and Reservoirs: Research \& Management 4: 101-105.

Quirós R., Rennella A. M., Boveri M. B., Rosso J. J., Sosnovsky A. 2002. Factores que afectan la estructura y el funcionamiento de las lagunas pampeanas. Ecología Austral 12: 175-185.

Reyna S., Reyna M., Orso M., Reyna E., Lábaque M., Gómez C. 2005. Plan de gestión de los recursos hídricos de la provincia de Córdoba. Subsecretaria de Recursos Hídricos. 
Rodríguez M. I., Granero I. M., Bustamante M. A., Avena M., Bonfanti E., Busso F., Girbal A. 2002. Análisis de la composición iónica de las aguas del embalse San Roque, Córdoba. XIX Congreso Nacional del Agua, Agosto Villa Carlos Paz, Córdoba, Argentina. Centro de la Región Semiárida (CIRSA).

Rodríguez M. I., Ruiz M., Vilchez G., Crema N., Ruibal Conti A. L., Bustamante M. A., Angelaccio C. M., Busso F., Bonfanti E., López F. 2010. Monitoreo Integral de un Cuerpo de Agua Eutrófico Embalse San Roque (Córdoba, Argentina). Actas I Congreso Internacional Sobre Gestión y Tratamiento del Agua. Córdoba, Argentina.
Rodríguez M. I., Ruiz M., Nadal F., Halac S., Olivera P., Busso F., Bonfanti E. 2013. Monitoreo y calidad de agua del embalse San Roque (Córdoba, Argentina). Actas del XXIV Congreso Nacional del Agua. San Juan. Argentina.

Roset J., Aguayo S., Muñoz M. J. 2001. Detección de cianobacterias y sus toxinas. Una revisión. Revista de Toxicología 18: 65- 71.

Tell G. 1985. Catálogo de las Algas de Agua Dulce de la República Argentina. Bibliotheca Phycologica 70. Vaduz: J. Cramer.

Tracanna B. C. 1985. Algas del Noroeste Argentino (excluyendo a las Diatomophyceae). Ópera Lilloana 35: 1-136.

Wenzel M. T., Díaz M. M. 2008. Cyanophyta del Parque Nacional Nahuel Huapi (Argentina). Darwiniana 46 (1): 51-65. 